\title{
The effect of differentiation via internal versus external magma oceans on the carbon and nitrogen budgets of rocky planets
}

\author{
DAMANVEER S. GREWAL ${ }^{* 1}$, RAJDEEP DASGUPTA ${ }^{1}$, \\ JAMES EGUCHI $^{2}$
}

${ }^{1}$ Department of Earth, Environmental and Planetary Sciences, Rice University, USA

${ }^{2}$ Department of Earth and Planetary Sciences, University of California Riverside, USA

*correspondence: dsg10@ rice.edu

The fate of major volatiles during the evolution of protoplanetary bodies and its subsequent effect on the volatile accretion history of rocky planets is poorly understood. Evidence for the widespread differentiation of the earliest formed planetesimals and planetary embryos makes their volatile inventories susceptible to fractionation between core, magma ocean (MO) and atmosphere (and subsequent loss to space). This is especially important for carbon (C) and nitrogen $(\mathrm{N})$, which may partition into any of the three reservoirs, albeit in different proportions depending upon the chemistry of MOs. Recent findings have shown that even the parent bodies of primitive chondrites did not escape differentiation such that their interiors, overlain by unmelted chondritic crusts, also underwent large-scale melting. Therefore, to track the evolution of $\mathrm{C}$ and $\mathrm{N}$ from primitive dust to present-day planets, it is important to constrain their fate during end-member protoplanetary differentiation regimes, i.e., internal, closed system MOs (MO-core fractionation) and external, open system MOs (atmosphereMO-core fractionation).

Here we present a thermodynamic modelling framework to track $\mathrm{C}$ and $\mathrm{N}$ fractionation between atmosphere, $\mathrm{MO}$ and core reservoirs as a function of the composition of their accreting material and sizes of the parent bodies. For external MOs, $\mathrm{C}$ and $\mathrm{N}$ in the MOs are calculated based on their vapor pressure-imposed solubility in the silicate melts while the exchange between MOs and core forming alloy melts for external as well as internal MOs are calculated using alloysilicate melt partition coefficients. For external MOs, 89-99\% of the accreted $\mathrm{C}$ and $\mathrm{N}$ inventories reside in the $\mathrm{MO}$ equilibrated atmosphere, $1-11 \%$ in their cores and less than $1 \% \mathrm{C}-\mathrm{N}$ in their MOs. Whereas for internal MOs, the cores are the major $\mathrm{C}$ and $\mathrm{N}$ bearing reservoir (90-99\%). Therefore, $\mathrm{C}-\mathrm{N}$ inventories of larger planets were affected by the relative prevalence of these end-member protoplanetary differentiation regimes. 